\title{
A LINKUP BETWEEN SEYFERT'S AND THEIR SURROUNDINGS
}

\author{
A.P. MAHTESSIAN ${ }^{1}$, E.YE. KHACHIKIAN ${ }^{1}$ AND H. TIERSCH ${ }^{2}$ \\ ${ }^{1}$ Byurakan Astrophysical Observatory, 378433 Byurakan, Armenia \\ ${ }^{2}$ Potsdam University, 14482 Potsdam, An der Sternwarte 16, \\ Germany
}

The observed physical characteristics of galaxies are (at least partly) determined by the influence of the surroundings after the formation of the galaxies. The examination of the frequency of the occurence of active galaxies, i.e. Seyfert galaxies, in different galaxy systems compared to a sample of field galaxies gives possibly an insight into this problem.

The galaxy groups were taken from the CfA redshift survey, the list of them is published in Mahtessian (1992, Soob. Byurakan. Obs. 65).

The investigated correlations are:

1. The occurence of Seyfert's in groups with different number of members: In the sequence going from single galaxies (i.e. "groups" with one member) to member-rich groups the relative number of Sy1 and Sy2 does not change, but the Sy3 are more often in galaxy groups than among the single galaxies. 2. The correlation between the morphological content of a group and the occurence of Seyfert's: The galaxy groups with and without Seyfert's do not distinguish from each other, i.e. the morphological content of a group has obviously no influence on the existence of Seyfert's within the groups. 3. The correlation between the density of galaxies in the groups and the occurence of Scyfert's: As a parameter of the density the mean pairwise distance between the galaxies is used. We could not find that the number of Seyfert's depends on the density of the galaxy groups.

4. The correlation between the velocity dispersion within galaxy groups and the occurcnce of Scyfert's: The groups with Seyfert's have in the mean a larger velocity dispersion than the groups without Seyfert's. This difference is statistically more significant for groups with Sy1 and Sy2.

5. The correlation betwecn the crossing time $\tau$ of the galaxy group and the availability of the Seyfert's: Groups with Seyfert's have in the mean a smaller crossing time than groups without Seyfert's. 